\title{
Cartografía de la reforma educativa en Chile. Mercado, competencia y mejoramiento escolar ${ }^{*}$
}

\author{
Juan Antonio Carrasco Bahamonde (D) \\ Daniel Alonso Carrasco Bahamonde"(D)
}

\section{RESUMEN}

La experiencia acumulada de reformas en educación chilena se caracteriza por haber depositado una confianza extrema en el mercado como contexto de regulación institucional. El mercado constituiría un ambiente propicio para detonar procesos de mejoramiento escolar, alcanzar resultados esperados e incrementar el bienestar de las familias. El debate en torno a si estas políticas contribuyen a elevar la calidad está en el centro del interés público. Consecuencias no deseadas serían la mercantilización del sistema, la erosión de la matrícula municipal y la segregación escolar. Atendiendo a esto, el presente artículo despliega una revisión de las estrategias conceptuales en torno a las políticas de mercado en educación, analizando sus componentes centrales y las acciones que las escuelas elaboran en ambientes competitivos.

\section{PALABRAS CLAVE}

cuasi-mercado; mejoramiento escolar; calidad de la educación.

\footnotetext{
*Agradecemos a CONICYT CHILE y el Programa de Becas de Estudios de Magíster (2012) su significativo apoyo durante el desarrollo de este trabajo.

'Universidad de Chile, Santiago, Chile.

"Universidad Tecnológica de Chile, Santiago, Chile.
} 


\title{
MAPPING OF THE EDUCATIONAL REFORM IN CHILE. MARKET, COMPETENCE AND SCHOOL IMPROVEMENT
}

\begin{abstract}
The accumulated experience of reforms in the Chilean education is characterized by having placed an extreme confidence in the market as a context of institutional regulation. The market would provide an favorable environment to detonating processes of school improvement, achieving expected results and increasing the well-being of families. The debate over whether these policies contribute to raising quality is at the center of the public interest. Unintended consequences would be the commodification of the system, the erosion of municipal tuition and school segregation. The present article presents a review of the conceptual strategies around market policies in education, analyzing their central components and the actions that schools develop in competitive environments.
\end{abstract}

KEYWORDS

quase-market; school improvement; quality of education.

\section{CARTOGRAFIA DA REFORMA EDUCACIONAL NO CHILE. MERCADO, COMPETÊNCIA E MELHORIA ESCOLAR}

\section{RESUMO}

A experiência acumulada de reformas na educação chilena caracteriza-se por ter colocado uma confiança extrema no mercado como um contexto de regulação institucional. $\mathrm{O}$ mercado proporcionaria um ambiente propício para detonar processos de melhoria escolar, alcançar os resultados esperados e aumentar o bem-estar das famílias. O debate sobre se essas políticas contribuem para o aumento da qualidade está no centro do interesse público. Consequências não intencionais seriam a mercantilização do sistema, a erosão das matrículas municipais e a segregação escolar. $\mathrm{O}$ presente artigo apresenta uma revisão das estratégias conceituais em torno das políticas de mercado na educação, analisando seus componentes centrais e as ações desenvolvidas pelas escolas em ambientes competitivos.

PALAVRAS-CHAVE

quase-mercado; melhoria escolar; qualidade da educação. 


\section{INTRODUCCIÓN}

La experiencia acumulada de reformas en educación chilena se caracteriza por haber depositado una confianza extrema en el mercado como marco regulatorio institucional, avanzando en el último decenio a formas de gobernanza en base a resultados y estándares de aprendizaje (Bellei y Vanni, 2015). La noción virtuosa del mercado en el diseño de sistemas educacionales supone que las escuelas responden progresivamente a la presión competitiva por captar recursos, identificando las señales que brinda un contexto social de libre elección (Friedman, 1955; Chubb y Moe, 1988). La institucionalización de los mercados educativos se asocia, en este sentido, a un tipo de políticas donde las familias tendrían mayor control sobre las escuelas y más posibilidades de influir en la educación de sus hijos, expandiendo el bienestar de aquellas familias que pueden elegir entre distintas alternativas educacionales.

Las políticas que incentivan la competencia entre escuelas suscriben los argumentos económicos sobre la naturaleza superior de los mercados. La presión competitiva que desata el mercado constituiría un ambiente propicio para detonar procesos de mejoramiento educativo, alcanzar la calidad esperada e incrementar el bienestar de las familias. En un diseño de gobernanza institucional de estas características es esperable que las escuelas presenten atributos como una fuerte orientación a las familias, una actitud estratégica y promocional en el ámbito de la competencia local y una creciente autonomía en sus definiciones programático-curriculares, lo cual constituiría, desde el punto de vista de sus defensores, elementos facilitadores de procesos de mejoramiento sostenido, tanto en las escuelas como a nivel sistémico (Chubb y Moe, 1988; Tooley, 1993).

Desde el punto de vista institucional, estos mercados educativos constituyen un ensamblaje de políticas de gobernanza que, a diferencia de un libre mercado clásico, integra múltiples mecanismos de regulación, estrategias de dirección e instituciones de gobierno. Los gobiernos establecen las "reglas del juego" mediante un marco de currículum nacional y sistemas de pruebas estandarizadas, entre otros mecanismos, que regulan e incentivan la competencia entre las escuelas. Por lo tanto, los "cuasi-mercados" educativos constituyen una forma de gobernanza de carácter híbrido que presenta elementos burocráticos de control, por un lado, y dinámicas asociadas a la privatización de los sistemas educativos, la competencia entre escuelas y la elección escolar de las familias, por otro (Van Zanten, 2005).

Basados en este esquema, a partir de 1981 la política educativa en Chile desarrolló reformas estructurales sentando las bases de una institucionalidad basada en el mercado. Esto incentivó la entrada de nuevos competidores privados a partir de un fuerte financiamiento público, presionando al conjunto de las escuelas por mejorar sus resultados, ampliar la cobertura escolar y ofrecer mayores alternativas a las familias. Siguiendo a Bellei (2007), mientras la matrícula de los establecimientos públicos representaba en 1981 el 78\% del total, en 1991 había disminuido su participación a un 58\%. Desde el 2006, la matrícula de los establecimientos privados subvencionados aumentó sostenidamente, pasando de 950 mil alumnos en 1991 a 1,6 millones en 2006; prácticamente la misma proporción de estudiantes de establecimientos públicos. De este modo, si en 1980 alrededor de un 15\% de los 
estudiantes se educaba en escuelas privadas, en 2014 se estima que lo hacía un 60\% (Gráfico 1) (Canales et al., 2016).

El debate en torno a si estas políticas contribuyen a elevar la calidad de la educación está en el centro del interés público (Tokman, 2002; Auguste y Valenzuela, 2004; Bellei, 2007). En perspectiva, el mejoramiento educativo en Chile posee un alcance parcelado, poco efectivo y de baja sostenibilidad (Bellei et al., 2014). Consecuencias no deseadas serán la excesiva mercantilización del sistema educativo, la erosión de la matrícula municipal y el aumento en la segregación escolar sin mejoramiento a nivel agregado del sistema educativo (Carnoy, 1998; Bellei et al., 2008; Cox, 2012). Actualmente, un nuevo ciclo de reforma educativa se plantea revertir los efectos que las fuerzas diferenciadoras del mercado desataron en la educación chilena, siendo la calidad y equidad de los aprendizajes las tensiones centrales del sistema educativo.

Atendiendo a este escenario, el presente artículo presenta una breve revisión de los contextos de política educativa y las estrategias conceptuales para abordar el fenómeno del mercado en la educación chilena, analizando sus componentes centrales y el tipo de respuestas que las escuelas elaboran en estos ambientes competitivos. El debate respecto a si las reformas de mercado contribuyen a elevar la calidad de la educación está en el centro del interés académico y resulta relevante comprender cómo se relaciona este contexto regulatorio con los procesos de mejoramiento escolar en un periodo de expansión y consolidación de la educación administrada por sostenedores privados en Chile (Tokman, 2002; Auguste y Valenzuela, 2004; Bellei, 2007).

\section{GLOBALIZACIÓN, CUASI-MERCADO EDUCATIVO Y POLITICAS DE BIENESTAR}

El proceso de globalización puede ser entendido como una tendencia a la generación de mayor interdependencia internacional e integración supranacional que se orienta a la reestructuración de las economías bajo los imperativos de la competitividad, el desarrollo de renovadas estrategias políticas y el incremento en

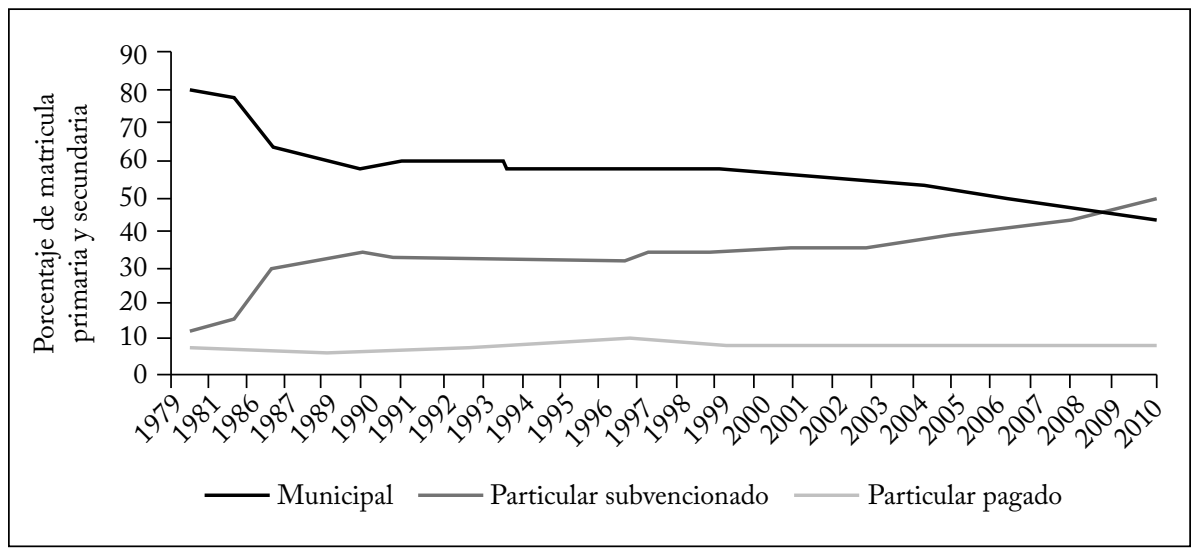

Gráfico 1 - Evolución matrícula escolar según dependencia (1979-2010).

Fuente: Elacqua y Santos (2013, p. 91). 
la conciencia nacional de comunidades locales (Van Zanten, 2005). De este modo, las fuerzas que estarían a la base de este proceso se asocian a la desindustrialización de la economía, la liberalización del comercio, el desarrollo de nuevas estrategias de gobierno, el cambio en los patrones demográficos y la re-organización de la estructura familiar, entre otras tendencias que contextualizan el trabajo de las escuelas.

Las sociedades contemporáneas explorarían nuevas formas de política social como respuesta a la crisis de legitimidad y eficiencia del Estado Burocrático Centralizado (Castells, 1997). El imperativo económico de la globalización consistiría en la competitividad, a nivel individual y agregado en economías cada vez más dinámicas e interdependientes, lo que desafía a los gobiernos desde el punto de vista de la generación de políticas sociales y replantea de manera crítica la cuestión del bienestar social. El énfasis en la competitividad desplazaría el eje de la política social clásica y presionaría a la generación de programas más "eficientes", en una relación de costo/beneficio, que muchas veces se traducen en paquetes menos " $g e$ nerosos" de política social.

Desde la gestión de los sistemas políticos, la noción de bienestar se orientaría a la reestructuración de las estrategias de gobierno que luchan por alcanzar la estabilidad democrática. Así, mientras que la política clásica de bienestar (siglo XX) fue un proceso progresivo de generación de bienes, servicios y beneficios sociales para una población creciente, la actual política de bienestar se orientaría cada vez más a criterios de costo/efectividad en la inversión pública, el ajuste del gasto social y el refuerzo de patrones de estratificación social orientados al mercado. Esto supone un conjunto de medidas que generan resistencia en sectores cuyo bienestar depende fuertemente del gasto social, incrementando los riesgos e incertidumbres en la esfera individual y privilegiando los equilibrios macro-económicos y la gobernabilidad política (Bauman, 2013).

En este escenario se consolida la masificación de los sistemas educativos, donde la educación primaria y secundaria se universalizan, expandiendo oportunidades de acceso a posiciones educativas y sociales ventajosas a mayores sectores, al tiempo que los grados escolares pierden, en términos relativos, su prestigio y poder diferenciador. En muchos países, la masificación de los sistemas educativos está acompañada de políticas privatizadoras orientadas a la promoción de grupos desfavorecidos, dado que, a pesar de la expansión de los años de escolaridad, las desigualdades no solo persisten, sino que se acentúan. ${ }^{1}$ Las formas de gobernanza

1 Van Zanten (2005) sostiene que este proceso puede afectar a distintos grupos desde el punto de vista de la estructura social y el acceso a la educación. Así, mientras en las elites existirían mayores recursos para aprovechar estas oportunidades, ya que tendrían una fuerte orientación a la internacionalización, el manejo de idiomas, el acceso a redes y una educación altamente selectiva, en las clases medias estas políticas de expansión pueden ser percibidas como un riesgo de clausura social. Las clases medias emergentes, que dependen más de recursos económicos para mantener y reproducir sus posiciones sociales, presionan a las escuelas por una educación más instrumental orientada al mercado, mientras que los grupos medios con mayor capital educativo tienden a demandar un concepto más sustantivo de educación. Contrariamente, las familias de bajos ingresos parecen menos habilitadas para capturar las oportunidades que pone en juego este proceso. 
institucional "post-burocráticas" constituyen, en este sentido, espacios de multi-regulación que integran dinámicas de mercado y mecanismos de gestión en base a resultados, rendición de cuentas y evaluaciones estandarizadas, entre otros dispositivos de política educativa, guiados por la idea que el incentivo a la competencia es un elemento dinamizador capaz de activar procesos de mejoramiento de la calidad y generación de resultados (Maroy, 2004; Van Zanten, 2005).

Basados en este esquema, la política educativa en Chile desarrolló a partir de 1981 las bases de una nueva institucionalidad basada en el mercado. De manera pionera, estas políticas introdujeron incentivos a la formación de escuelas administradas por sostenedores privados, incluyendo una subvención pública mediante un sistema de financiamiento per cápita a la demanda de carácter universal, una política de igual trato entre escuelas públicas y particulares subvencionadas, una descentralización de la gestión educativa anclándola en los gobiernos locales y la implementación de pruebas estandarizadas para medir aprendizajes en los estudiantes chilenos. Esto incentivó la entrada de competidores privados a partir de un fuerte financiamiento público, presionando al conjunto de las escuelas por mejorar sus resultados, ampliar la cobertura escolar y ofrecer mayores alternativas a las familias. ${ }^{2}$

En 1990 se resitúa en el centro de la agenda pública los objetivos de equidad y calidad de la educación, aunque con fuertes restricciones constitucionales y políticas. El espíritu reformista que inaugura este periodo no solo no apuntará a modificar las bases institucionales del sistema educativo, sino que incluso profundizará elementos del ciclo de políticas anterior como, por ejemplo, la incorporación del financiamiento compartido en 1993. En lo esencial, los gobiernos democráticos mantendrán un tratamiento similar en cuanto a los subsidios escolares y el régimen de "igual trato" entre escuelas públicas y particulares subvencionadas (Bellei et al., 2010). A partir de estas reformas, el sistema educativo presentará principalmente tres tipos de escuelas: escuelas públicas administradas por municipios, escuelas particulares subvencionadas con fondos públicos y escuelas particulares financiadas por el pago de las familias.

Como contrapunto, en la década del 2000 la opinión pública adquiere conciencia crítica respecto de los límites de la reforma educativa y la sostenibilidad de la estrategia global se torna problemática. Comienza a formarse un interés creciente en los "impactos" de las políticas educativas, mientras la evaluación de los resultados parece corroborar una sensación pesimista (Delanoy, 2000; Tokman, 2002; Bellei y Vanni, 2015). La lección de política educativa fue asimilada como un déficit de regulación por parte del gobierno: el mercado en su forma pura no resolvería las cuestiones de calidad y equidad, requiriendo mayores controles y claridad de roles.

2 Si bien la participación de instituciones privadas en la provisión escolar no constituye un elemento nuevo en la historia educativa chilena, existiendo una tradición de escuelas privadas, principalmente de carácter confesional, orientada a la formación de las elites políticas e intelectuales, las reformas de mercado en educación implementadas modificaron profundamente el contexto regulatorio institucional radicalizando elementos que estaban presentes en la institucionalidad educativa, aunque esta vez adquieren una relevancia central irradiando al conjunto del sistema educativo. 
Con la formación del Sistema de Aseguramiento de la Calidad y la Ley General de Educación (Gobierno de Chile, 2009) el escenario nacional tiende a la conformación de un "cuasi-mercado" corregido, donde la nueva política educativa apuntará al fortalecimiento de instrumentos de rendición de cuentas y la institucionalidad pública como sustrato del mejoramiento educativo.

\section{ONTOLOGÍA DEL MERCADO: COMPONENTES DE LA REFORMA EDUCATIVA}

Ya sea que los actores privados colaboren en términos específicos mediante insumos y/o soporte al proceso educativo, o que los gobiernos establezcan contratos con organizaciones privadas para que administren escuelas, es posible encontrar distintos modelos de privatización. Actualmente, a nivel global, la estrategia de participación mixta más extendida promueve políticas que destinan financiamiento público a la creación de escuelas privadas para incentivar la competencia y expansión de alternativas educacionales (Patrinos et al., 2009).

Con un sector privado ampliamente desarrollado destacan las experiencias de Bélgica y Holanda, mientras que los casos de Estados Unidos, Canadá y Colombia responden a la introducción de mecanismos de mercado en un marco flexible que permite a sostenedores privados administrar escuelas públicas existentes. En Inglaterra se observa un enfoque más orientado al mercado con un fuerte sistema de rendición de cuentas, mientras que en Suecia se advierte un sistema de financiamiento per cápita a la demanda universal ("vouchers") que incentiva la creación de escuelas privadas de manera similar a la experiencia chilena, aunque restringiendo el co-pago de las familias (Bellei y Trivelli, 2014).

En perspectiva, las políticas de mercado en Chile profundizan elementos que estaban presentes en el sistema educativo - por ejemplo, la participación de escuelas privadas de tipo religioso - , aunque, esta vez, supeditando el sistema a una lógica basada en la competencia entre escuelas. La especificidad de la experiencia chilena residiría en la radicalidad con que se implementa este tipo de política, la visión virtuosa de los impactos que tendría en el bienestar de las familias, el alcance geográfico que posee y la maduración de más de tres décadas de esta experiencia de política educativa. De este modo, las reformas de mercado en educación chilena se asocian a la expansión de la provisión privada de servicios educacionales, la introducción de mecanismos de competencia entre escuelas y la expansión de las posibilidades de familias de elegir la escuela de sus hijos (Bellei, 2007).

\section{PRIVATIZACIÓN DE LOS SISTEMAS EDUCATIVOS}

La privatización de los sistemas educativos constituye un fenómeno complejo que se expande durante las últimas décadas y responde a políticas inspiradas en un contexto de influencia neoliberal. Esta tendencia sería influida por procesos como la transformaciones de los modos de producción y consumo post-fordista que presionan a los sistemas educativos a elevar su competitividad, la crisis de las 
visiones de planificación centralizada y provisión de servicios sociales por parte del Estado, el ajuste del gasto social que exacerba la demanda de eficiencia y la ansiedad de estatus que lleva a los "grupos medios" a asumir una actitud estratégica respecto la educación de sus hijos (Maroy, 2004).

Para Bellei y Orellana (2014), el concepto de privatización encierra una complejidad que es subestimada y su uso en el debate público tiende a ser por lo menos difuso. Existirían distintas dimensiones susceptibles de ser privatizadas en los sistemas educativos: provisión de insumos y servicios educacionales, modelos de gestión educativa y/o relación con las familias y padres (electores). Los autores distinguen, en este sentido, entre la privatización interna de la educación pública y aquello que denominan privatización abierta de la educación, orientada a la expansión de la provisión escolar privada. Así, las políticas privatizadoras pueden estar motivadas por distintas razones, siendo sus implicancias políticas diversas (Bellei y Orellana, 2014).

En primer lugar, la privatización se puede articular a partir de la valoración del rol público de la educación que imparten establecimientos privados, siendo actores auxiliares de un sistema donde el componente dominante lo constituye la educación pública. A diferencia de lo anterior, un enfoque propiamente de mercado sostiene que la privatización no solo colabora con la provisión pública en sectores específicos, sino que permite elevar la eficiencia, efectividad y calidad mediante la competencia entre escuelas, introduciendo una mejora a nivel agregado y permeando la organización interna de las escuelas. Finalmente, en tercer lugar, encontramos un tipo de privatización que se organiza en torno a la necesidad de apoyar la expansión de la cobertura en áreas geográficas de difícil acceso o que cuentan con demanda suficiente, no teniendo el Estado la capacidad de ampliar los servicios educacionales al conjunto de la población.

Ball y Youdell (2008) distinguen entre una privatización endógena y exógena de la educación pública. La "endógena" consistiría en la aplicación de tecnologías y diseños de gestión que proyectan sobre la escuela un ethos empresarial orientado a la eficiencia y calidad en resultados y procesos, mediante la generación de unidades autónomas en la escuela, una orientación a la rendición de cuentas y/o incentivos económicos asociados al rendimiento. La "exógena" guarda relación con la apertura de la escuela a intereses económicos más amplios como la generación de utilidades, contratos de venta de servicios, participación de agentes privados que compiten por la matricula, alianzas con el sector productivo o competencia en el ámbito local, lo que no constituye solo un cambio tecnológico en las escuelas, sino una nueva configuración de roles, valores e identidades que re-significan el sentido de la comunidad educativa y sus miembros.

De este modo, lo específico de un modelo de gobernanza institucional basado en el mercado es una privatización creciente que incentiva la participación de privados y la competencia entre escuelas como medio para generar procesos de mejoramiento sistémico. Estudios respecto a la efectividad de las políticas de mercado en Chile han puesto en cuestión la tesis de que las escuelas privadas subvencionadas contribuirían a mejorar las escuelas públicas a través de un efecto de competencia, advirtiendo que la expansión de la educación privada subvencionada no habría pro- 
vocado un mejoramiento apreciable de la calidad del sistema escolar chileno como un todo, aumentado, como contrapartida, la segmentación y la inequidad (Bellei, 2007). En este contexto, las escuelas experimentan una tensión que las presiona a relevar símbolos de estatus, desarrollar actitudes y atributos cercanos al marketing, la innovación parcial y la competitividad.

\section{EXPANSIÓN DE LA COMPETENCIA ENTRE ESCUELAS}

Asociado al supuesto de la naturaleza superior de los mercados en los procesos de mejoramiento escolar, un segundo supuesto a la base de los cuasi-mercados educativos sostiene que las instituciones escolares reaccionarían ante la presión competitiva del entorno por optimizar su eficiencia y efectividad en la generación de aprendizajes, situando en el centro el perfil de preferencia y elección de las familias (Chubb y Moe, 1988; Tooley, 1993). La presión competitiva que desata el mercado liberaría la energía creativa de los actores, en analogía a la actividad empresarial, estimulando la innovación y búsqueda de nuevas respuestas y soluciones.

Así, la competencia entre escuelas contribuiría a diseminar la calidad en los sistemas educativos en una relación costo/oportunidad beneficiosa para la política social, especialmente en países en vías de desarrollo y/o en contextos de ajuste económico. La información confiable brindada por los reguladores del sistema instituciones de gobierno, ente otros - permitiría a los padres tomar decisiones en base a criterios de calidad, lo que detonaría la diversificación e innovación pedagógica, la optimización en la gestión de recursos públicos y el mejoramiento sostenido en las escuelas.

Por lo tanto, la competencia contribuiría a diseminar la calidad e incentivar la diferenciación horizontal de la "oferta formativa", guiándose según las señales que brinda el contexto social y ampliando las oportunidades de elección y bienestar de las familias. Las escuelas que reciben recursos públicos serán financiadas por un subsidio per cápita que "sigue al alumno" (voucher), de forma que las escuelas deben competir por captar las preferencias de las familias (Canales et al., 2016).

Existe una vertiente de investigación crítica respecto la capacidad que tiene el mercado de modelar las respuestas de las escuelas. Para Verger et al. (2016), la competencia en contextos de mercado no promueve necesariamente la calidad, pudiendo incentivar el desarrollo de estrategias para atraer estudiantes mejor dispuestos al logro escolar por parte de establecimientos particular subvencionado. Otro factor crítico sería la erosión de la confianza hacia las escuelas, la estigmatización de la educación pública y el incremento de la segregación socio-económica del sistema educativo. Este argumento sostiene que el mercado profundizaría la segregación socio-económica de los territorios, las desigualdades presentes en la sociedad y el debilitamiento de la cohesión social (Bellei et al., 2008).

Cómo los procesos de mejoramiento se dificultan o facilitan dadas las dinámicas de mercado en el sistema educativo chileno va a depender fuertemente de cómo se insertan y relacionan en un contexto específico de competencia local. Para Lundström y Parding (2011), la competencia no debe ser simplemente asimilada a la dicotomía entre escuelas públicas y privadas, sino que estaría fuertemente 
sujeta a la configuración específica que ésta adquiere en un territorio o espacio de interdependencia. Del mismo modo, en un contexto de cuasi-mercado, la lógica de la competencia es extensiva al conjunto de escuelas, por lo que no son solo las escuelas privadas las que desarrollan estrategias de posicionamiento en un territorio, aunque en el caso chileno parecen más proclives a ello.

\section{POLÍTICAS DE ELECCIÓN ESCOLAR}

Una tercera dimensión que está a la base de las reformas orientadas al mercado sostiene que la preferencia de las familias en un sistema de elección abierta, y no de asignación territorial u otro mecanismo intermedio, aumentaría el bienestar de las familias permitiendo llevar a sus hijos a establecimientos de mejor calidad (Carnoy, 1998). La institucionalización de los cuasi-mercados educativos se asocia, en este sentido, a un tipo de políticas donde las familias tendrían mayor control sobre las escuelas y más posibilidades de influir en la educación de sus hijos (Atria, 2012).

Desde esta perspectiva, los padres priorizarían la calidad de la enseñanza y revelarían sus preferencias a los proveedores mediante la elección, teniendo acceso a información suficiente para monitorear la calidad y ejercer voz en las escuelas. Los gobiernos ofrecerían información sobre la calidad de las escuelas, al tiempo que estas se enfocan en estrategias para mejorar activamente la enseñanza. Esto supone, además, la posibilidad de elegir escuelas a través de un sistema de "vouchers" y una distribución territorial normal de proyectos educativos de calidad a nivel regional y comunal. Las escuelas de bajo desempeño desaparecerían progresivamente por falta de demanda o decisión política.

En un contexto de mercado la evaluación de la escuela estaría más supeditada a la generación de aprendizajes efectivos, y, por lo tanto, en línea con los intereses directos de los padres. Familias y estudiantes tendrían una participación mayor en las definiciones programáticas de la escuela, su cultura y grado de especialización. De este modo, un diseño institucional orientado al mercado descansaría más en los recursos pedagógicos, institucionales y competencias profesionales de la escuela y, en definitiva, en la capacidad efectiva de generar aprendizajes para los estudiantes (Chubb y Moe, 1988).

Ahora bien, la evidencia empírica pareciera sugerir que los efectos agregados de la elección escolar en lugar de tener un impacto relevante en los logros académicos de los estudiantes, habría tenido efectos negativos en términos de segregación social. Como señalan Canales et al. (2016, p. 89-91):

Las familias deciden orientándose esencialmente por razones sociales, procurando separarse de grupos socioeconómicamente inferiores (considerados riesgosos), lo cual les hace descartar escuelas públicas, gratuitas y no selectivas [...] La evidencia sobre los efectos agregados de la elección escolar sugiere que no habría tenido un impacto relevante en los logros académicos de los alumnos, pero sí efectos negativos en términos de la segregación social y racial de las escuelas [...] Los estudios también han mostrado diversidad en las prácticas de elección escolar de las familias. Esta diversidad no se reduce a desigualdades de recursos 
económicos, sino que se asocia con diferentes posiciones en la estructura social y configuraciones culturales, especialmente de clase social, raza, etnia y lengua.

Entre los elementos que afectan la elección, se advierte que las escuelas tienden a ser asimiladas a su entorno en términos de estigmatización o prestigio: escuelas emplazadas en territorios que enfrentan problemáticas sociales tienden a ser percibidas como escuelas complejas, constituyendo una suerte de caja de resonancia de los conflictos y la criticidad socio-económica de su entorno. Asimismo, mientras las familias de bajos ingresos privilegian la distancia como principal variable, los contextos urbanos ofrecen un espectro mayor de posibilidades en desmedro de los rurales. En este sentido, la política de elección escolar sería geográficamente ingenua, siendo factores como el radio de distancia entre el establecimiento y el hogar o la distribución territorial de la oferta elementos diferenciadores que actúan sobre el espectro de posibilidades según estratificación socio-económica de las familias (Flores y Carrasco, 2013).

\section{RESPUESTAS DE LAS ESCUELAS COMO LÓGICAS DE ACCIÓN COMPLEJAS}

De este modo, cómo la competencia afecta a las escuelas y las respuestas que estas elaboran variará significativamente de acuerdos a elementos contextuales como sus dinámicas territoriales, demográficas, políticas y sociales. Maroy (2004) sostendrá, a partir de una investigación comparativa en distintas zonas geográficas y diseños institucionales, que es posible observar la formación de diversos clúster o espacios de interdependencia competitiva entre escuelas en el espacio local. La intensidad y significación de la competencia variaría según el territorio, sus políticas y las lógicas de acción que desarrollan las escuelas.

Siguiendo a Van Zanten (2005), los espacios de interdependencia competitiva se estructuran a partir de distintos niveles de acción, incluyendo el marco regulatorio y las políticas nacionales, las directrices de los agentes regulatorios intermedios, las dinámicas demográficas o territoriales y las características e historia de las escuelas que forman parte de un espacio de interdependencia. Las lógicas de acción de agentes intermedios pueden variar de un espacio a otro, permeando la forma en que los marcos regulatorios son asimilados en cada espacio y escuela. ${ }^{3}$

3 En los segmentos medio-altos de un espacio local jerarquizado es más probable que las lógicas de acción se muevan en el polo agresivo ("emprendedor"), a partir del cual las escuelas desarrollan de manera activa estrategias que buscan captar a nuevos y más exigentes segmentos. En la sección baja de una jerarquía, las escuelas muchas veces no pueden sino asimilarse, de manera pasiva o activa, a los contextos socio-territoriales de exclusión y estigmatización en los cuales se insertan. Escuelas en zonas medias de la jerarquía local se esfuerzan por no perder el estatus ganado, mejorando la imagen que proyecta al exterior. Asimismo, algunas escuelas buscarán atraer y retener cierto tipo de estudiantes, evitando aquellos ligados a problemas socio-conductuales. Dado lo anterior, las escuelas resultan distintamente atractivas para las familias dentro de un espacio de interdependencia, destacando factores como la cercanía, los resultados y/o el clima escolar. 
Basándose en la experiencia de Francia y Bélgica, Van Zanten (2005) señala que las escuelas que han permanecido por periodos prolongados en posiciones de privilegio en la jerarquía local no perciben grandes incentivos a desarrollar estrategias de competencia agresiva ni tampoco en modificar sustancialmente su "oferta", conservando incluso programas altamente tradicionales de estilo clásico y academicista, mientras que una fracción de las elites en estos países opta activamente por una formación internacionalista, bilingüe y "modernista", a partir de la formación de nuevas escuelas orientadas a una educación selectiva que compite con instituciones de elite tradicionales.

Para Maroy (2004), la interdependencia basada en la competencia se genera a partir de la integración de la capacidad de las escuelas que tienen de atraer a las familias de acuerdo a criterios internos y externos. Mientras que entre los primeros destaca el clima escolar, el tamaño de la clase y el trabajo pedagógico de los profesores, entre los segundos está la promoción que realiza la escuela, las prácticas asociadas a la selectividad que ejercen y las acciones que desarrollan las autoridades locales en la concreción de sistemas regulatorios o iniciativas de política nacional. Sería precisamente en este doble movimiento donde se configuran las posibilidades de las escuelas por triunfar en contextos competitivos.

De este modo, las lógicas de acción de las escuelas no se derivan mecánicamente de los factores contextuales que constriñen o facilitan procesos en las escuelas. La coherencia entre las condiciones externas/internas y la lógica de acción de la escuela no es un fenómeno "natural" que se organice a partir de una acción adaptativa por parte de la escuela a condiciones del entorno. Se trata de un proceso político complejo y activo donde el rol de los directivos es precisamente construir y sostener una cierta coherencia y cohesión interna. Por supuesto, un cambio en el contexto inmediato de la escuela puede tender a modificar su lógica de acción, aunque su flexibilidad en las formas en que esta responde a contextos dinámicos da cuenta de sus posibilidades de perdurar en el mercado.

\section{MEJORAMIENTO ESCOLAR EN CONTEXTOS DE MERCADO}

Bellei y González (2013) caracterizan el mejoramiento escolar sostenido como un proceso de cambio multidimensional que afecta diversas áreas de trabajo de la escuela y la pedagogía, impulsado por un trabajo colectivo basado en la participación de los docentes y en colaboración activa con los equipos directivos. El mejoramiento genuino constituye una transformación de los modos de hacer y pensar de los docentes en la escuela, modificando la visión de los actores e institucionalizado en prácticas recurrentes, asumidas colectivamente por una comunidad educativa.

La institucionalización de estos procesos remite al despliegue de estrategias de gestión pedagógico-curricular donde los aprendizajes van tomando el centro de las preocupaciones institucionales y mejoran el desempeño "medio" de los docentes. En las escuelas que mejoran existe una claridad en los distintos roles de la organización escolar, se genera y gestiona periódicamente información sobre resultados académicos, alentando la formación de comunidades de aprendizaje donde los 
docentes aprenden de sus pares, conforman una identidad común, un fuerte sentido de propósito y pertenencia respecto a la institución (Bellei et al., 2014).

Siguiendo estos planteamientos, se observan distintas rutas de mejoramiento en escuelas chilenas entre los años 2000-2010 en una línea de progresión que va de impulsos parciales orientados a resultados en pruebas estandarizadas a niveles de mayor densidad e institucionalización de procesos de mejoramiento sostenido (Bellei et al., 2014). En este sentido, la tipología o rutas de mejoramiento que a continuación se presenta posee un carácter descriptivo y ayuda a esquematizar la complejidad de estos procesos, considerando como parámetros de entrada dimensiones como el nivel de institucionalización de las estrategias de mejoramiento, la sostenibilidad del proceso, su integralidad en los distintos niveles de la escuela como también cómo interactúan estos procesos con su cultura e identidad. Asimismo, existen desafíos exigentes y diferenciados en el pasaje de un ciclo a otro del mejoramiento genuino, entendido como la transformación de los modos de acción y pensamiento que tienen lugar en la escuela (Cuadro 1).

Ahora bien, las variables operacionales críticas para las escuelas en un entorno competitivo serán atraer y retener una matrícula de estudiantes suficiente para garantizar su funcionamiento y alcanzar resultados aceptables en las pruebas SIMCE. Ambos tipos de metas son comprometidas cada año entre sostenedores y escuelas, siendo monitoreados y abordados por ambos estamentos. Cuánto se expresa la presión competitiva que ejerce el entorno en el trabajo de las escuelas va a depender en buena parte de la posición relativa que adquieren frente a ambas dimensiones críticas de su trabajo.

Si bien existiría una dinámica de arrastre entre matrícula y resultados, la competencia entre las escuelas en Chile también estaría permeada por un "efecto reputacional" difícil de predecir considerando solo estos aspectos. Habría escuelas que, a pesar de obtener buenos resultados, no logran remontar su matrícula, como también escuelas que, sin obtenerlos, captan la preferencia de las familias. El prestigio que logran adquirir las escuelas no depende, en este sentido, solo de estos factores y se asocia fuertemente a la dependencia institucional, siendo las escuelas municipales afectadas por un escenario de desprestigio creciente: escuelas municipales tenderían a perder matrícula independiente de sus resultados, mientras escuelas particulares subvencionada la ganan incluso en ausencia de éstos (Weinstein et al., 2013).

Para Weinstein et al. (2013), esta complejidad se puede abordar a partir de cuatro escenarios posibles:

- escuelas exitosas, donde la escuela logra consolidar una situación de buenos resultados en las pruebas y goza de una matrícula suficiente;

- escuelas críticas, donde la escuela lucha por revertir los bajos resultados y la pérdida progresiva de matrícula;

- escuelas en escenarios de desprestigio, donde, a pesar de obtener buenos resultados en términos relativos, debe lidiar con el éxodo de las familias; $y$

- escuelas en escenarios reputacionales, donde, a pesar de no alcanzar los resultados esperados, la escuela mejora consistentemente su matrícula. Estos escenarios serían las coordenadas básicas desde donde las escuelas se sitúan frente a la presión competitiva que les plantea el mercado educativo chileno. 
Las metas, estrategias de acción y gestión de recursos de las escuelas van a depender del escenario que enfrenta cada una. Entre las estrategias para captar matricula, se destaca crear niveles pre-escolares que permitan anticipar los procesos de admisión y generar "semilleros", fidelizar un público objetivo reforzando la identidad territorial, consolidar el estatus diferenciado de las familias que acceden a la escuela, fortalecer la reputación de la escuela a partir de aspectos extra-académicos y difundir los resultados obtenidos entre las familias. Entre las estrategias para

Cuadro 1 - Tipología de rutas de mejoramiento educativo en Chile (2000-2010).

\begin{tabular}{|c|c|c|}
\hline $\begin{array}{l}\text { Tipo de } \\
\text { mejoramiento escolar }\end{array}$ & Orientación & Descripción \\
\hline Institucionalizado & $\begin{array}{c}\text { Construcción de } \\
\text { capacidades }\end{array}$ & $\begin{array}{l}\text { Alta normalización de los procesos en la escuela } \\
\text { y mejoramiento sostenido en periodos largos } \\
\text { de tiempo. Formación de comunidades de } \\
\text { aprendizaje donde se refuerza el trabajo colectivo } \\
\text { docente, la reflexividad y la observación de } \\
\text { prácticas. Carácter integral del mejoramiento } \\
\text { comprende distintos ciclos y sectores del } \\
\text { currículum. Alta institucionalización de las } \\
\text { estrategias de gestión, cultura escolar orientada a } \\
\text { la mejora y equipos cohesionados. }\end{array}$ \\
\hline Vía de institucionalización & $\begin{array}{l}\text { Respuesta } \\
\text { estratégica }\end{array}$ & $\begin{array}{l}\text { Presenta rasgos propios de escuelas efectivas } \\
\text { (uso intensivo de tiempo, disciplina y } \\
\text { convivencia, etc.) y un mejoramiento } \\
\text { sostenido, aunque todavía parcial. Foco en } \\
\text { el aprendizaje y estrategias de mejoramiento } \\
\text { del trabajo docente en proceso de } \\
\text { institucionalización. Sentido de identidad y } \\
\text { pertenencia a la escuela. Mejoramiento en } \\
\text { ciertos ciclos o sectores, fuertemente basado } \\
\text { en el trabajo directivo. }\end{array}$ \\
\hline Incipiente & Respuesta táctica & $\begin{array}{l}\text { Se observa una fase inicial de cambio a partir } \\
\text { de una reestructuración organizacional liderada } \\
\text { por directivos como respuesta a un sentido de } \\
\text { crisis institucional. Foco en los aprendizajes } \\
\text { y capacidades docentes, a partir de acciones } \\
\text { específicas que integran aspectos estratégicos del } \\
\text { mejoramiento. Orientación al trabajo colectivo } \\
\text { basado en la capacidad de liderazgo de directivos. } \\
\text { Mejoramiento orientado a aprendizajes básicos y } \\
\text { una institucionalización incipiente. }\end{array}$ \\
\hline Puntual & $\begin{array}{l}\text { Acciones específicas } \\
\text { de mejoramiento }\end{array}$ & $\begin{array}{l}\text { Heterogeneidad en las fuentes y acciones en la } \\
\text { base de estos procesos de mejoramiento. Baja } \\
\text { densidad del proceso de mejoramiento y fuerte } \\
\text { ordenamiento de la gestión y cultura de la escuela } \\
\text { en base resultados en pruebas estandarizadas. } \\
\text { Ausencia de un relato estructurado de identidad y } \\
\text { cultura compartida en la escuela. }\end{array}$ \\
\hline
\end{tabular}

Fuente: Bellei et al. (2014, p. 10). 
obtener resultados, se observa la selección de estudiantes privilegiando un perfil favorable al logro, un trabajo diferenciado hacia estudiantes que presentan rezago en sus aprendizajes, una priorización de los tiempos en los sectores evaluados, un fortalecimiento de las competencias docentes requeridas, la adopción de metodologías específicas para los sectores evaluados y la generación de incentivos a docentes para el cumplimiento de objetivos (Weinstein et al., 2013).

Estas estrategias pueden estar asociadas entre sí o situarse en escenarios específicos. En este sentido, existen estrategias difundidas entre escuelas para lograr sus metas independientes de su escenario específico como, por ejemplo, la adopción de metodologías específicas por sector, el trabajo diferenciado a estudiantes con dificultades de aprendizaje, la preparación para pruebas estandarizadas y la difusión de resultados académicos. Contrariamente, hay estrategias que se asocian directamente a los escenarios base que enfrentan las escuelas. Ciertamente, las escuelas municipales poseen un repertorio más acotado de acciones respecto a establecimientos particular subvencionados, los cuales cuentan con mayores posibilidades de maniobra, especialmente a la hora de establecer incentivos a los docentes para obtener resultados esperados (Weinstein et al., 2013).

Ahora bien, las fuentes de estatus no ligados a lo académico, en sentido tradicional, refieren a aspectos de infraestructura, implementación de la Jornada Escolar Completa, propuestas formativas complementarias (inglés, computación, etc.) y actividades extra-escolares asociadas a prácticas deportivas, artísticas y/o culturales. Según Weinstein et al. (2013, p. 245), la estrategia de consolidación del estatus de la escuela estaría íntimamente conectada con las políticas de selección que ejerce:

[...] la búsqueda de potenciar una identidad especial para quienes asisten a una cierta escuela (como mecanismo de reclutamiento de matrícula) se conecta con la selección académica que puede hacerse del estudiantado (como medio de subir los resultados académicos), reforzando el carácter exclusivo del establecimiento.

Siguiendo a Woods et al. (1998), los desafíos de las escuelas en un contexto de mercado, donde estas ofertan a comunidades que poseen alternativas de elección escolar, se pueden organizar a partir de una tensión permanente entre, por un lado, la elección de los padres, la diversidad curricular y la autonomía escolar, y, por otro, los valores de equidad e integración del sistema educativo. En este contexto, las estrategias que elaboran las escuelas en ambientes de elección y competencia pueden organizarse en dos niveles: acciones de tipo operacional y sistémico.

En el plano operacional, encontramos estrategias y actitudes del siguiente tipo:

- exploratorio, desarrollo de acciones orientadas a captar las señales del mercado educativo local y las tendencias de elección de los padres; y

- promocionales, las escuelas parecen especialmente preocupadas de proyectar una imagen positiva a su entorno desarrollando acciones específicas orientadas a reforzar su identidad y publicidad.

En el plano sistémico, encontramos estrategias orientadas a modificar un ámbito de funcionamiento de la organización escolar: 
- cambios sustantivos, acciones orientadas a modificar condiciones de trabajo de la escuela (currículum escolar, criterios de admisión, infraestructura, estilo de gestión, etc.);

- cambios estructurales, acciones de cambio orientadas a aspectos como la dependencia institucional, propiedad o financiamiento de la escuela; $y$

- cambios de administración de recursos, acciones concernientes a asegurar el presupuesto requerido, incluyendo, la búsqueda de financiamiento alternativo a la subvención regular por estudiante (Cuadro 2).

\section{CONCLUSIONES}

A lo largo del presente artículo hemos intentado comprender las nuevas formas de regulación de los sistemas educativos en contextos post-burocráticos y conocer cómo las características institucionales influyen en las lógicas de acción que desarrollan las escuelas en contextos de competencia. Las teorías de mercado asumen que la mayor efectividad depende del tipo de respuestas que elaboran las escuelas respecto a los incentivos y riesgos que pone en juego la competencia y el contexto social de elección que ésta propicia.

La perspectiva teórica sugiere que los modelos de gobernanza institucional post-burocráticos constituyen espacios de multi-regulación que integran fuerzas de mercado y mecanismos de gestión guiados por la idea que la competencia es un elemento dinamizador capaz de activar procesos de mejoramiento escolar. Por otro lado, algunas líneas de investigación vinculan críticamente los procesos de privatización a una mercantilización excesiva que erosiona la base de confianza hacia los sistemas escolares, a un constreñimiento de los poderes discrecionales de docentes y directivos, a una diseminación de una cultura gerencial que reduce el trabajo pedagógico a criterios

Cuadro 2 - Estrategias de las escuelas en contextos de competencia.

\begin{tabular}{|l|c|c|}
\hline $\begin{array}{l}\text { Dimensiones } \\
\text { estrategias escuela }\end{array}$ & Tipo & Definición \\
\hline Exploratorio & Operacional & $\begin{array}{c}\text { Desarrollo de acciones orientadas a captar las señales del } \\
\text { mercado educativo local y las tendencias de elección de } \\
\text { los padres (entrevistas, estudios, etc.). }\end{array}$ \\
\hline Promocional & Operacional & $\begin{array}{c}\text { Las escuelas proyectan una imagen positiva a su entorno } \\
\text { para lo cual desarrollan acciones específicas orientadas } \\
\text { reforzar su identidad y orientadas a la publicidad. }\end{array}$ \\
\hline Sustantivo & Sistémico & $\begin{array}{c}\text { Acciones orientadas a modificar condiciones de trabajo } \\
\text { de la escuela tales como el currículum escolar, criterios } \\
\text { de admisión, infraestructura, estilo de gestión, etc. }\end{array}$ \\
\hline Estructural & Sistémico & $\begin{array}{c}\text { Cambios orientados a aspectos como la dependencia } \\
\text { institucional, propiedad o financiamiento de la escuela. }\end{array}$ \\
\hline Administrativo & $\begin{array}{c}\text { Acciones concernientes a asegurar el presupuesto } \\
\text { requerido, incluyendo, la búsqueda de financiamiento } \\
\text { alternativo a la subvención regular por estudiante. }\end{array}$ \\
\hline
\end{tabular}

Fuente: Woods et al. (1998, p. 13). 
de costo/efectividad y a una segregación creciente del sistema educativo. Se advierte, en este sentido, que las políticas que exacerban la competencia parecieran descuidar los objetivos de inclusión, integración y cohesión social, sin incorporar necesariamente un mejoramiento en la calidad de los aprendizajes.

Esto pone de manifiesto que las políticas educativas no pueden simplemente articularse a partir de claves de efectividad escolar o referencias a "modelos" difíciles de ser implementadas en una trayectoria específica de cambio y mejoramiento educativo. Las escuelas desarrollan estrategias y modos diferenciados de procesar estos elementos contextuales dependiendo de su emplazamiento en la jerarquía local, sus atributos institucionales o la percepción de las comunidades; mientras en un extremo habría escuelas que logran beneficiarse de un ambiente de mercado sintonizando una demanda creciente de las familias, otras experimentan tensiones y desafíos permanentes para sostener su mejoramiento.

La elección escolar de padres en contextos de expansión urbana, con redes de transporte y servicios adecuados, no exacerba intrínsecamente la segregación socio-económica o inequidad en la distribución de oportunidades educacionales. No obstante, cuando se combina la elección con la libertad de oferta de escuelas que compiten, mediante la selección y admisión, por mayor cantidad de estudiantes y/o por captar aquellos que presentan mejores condiciones para el logro escolar, se reforzaría una dinámica de diferenciación y segregación que repercute en la conformación de un circuito de desigualdad de oportunidades para estudiantes socialmente desventajados y sus respectivas familias.

En este contexto, la actualidad de la política educativa remite a un panorama complejo; existe una expansión creciente de las oportunidades educativas, donde los padres han aumentado su capacidad de influir en la educación de sus hijos, no obstante, esto no ha resuelto los problemas asociados a la calidad y las desigualdades, profundizando, en muchos casos, la segregación educativa, económica y social existente en los territorios. La pregunta crítica no será si los cuasi-mercados educativos funcionan en un plano general, sino más bien sí efectivamente tienen la capacidad de modelar el comportamiento de los actores involucrados presionando hacia la calidad integral del sistema.

\section{REFERENCIAS}

Atria, F. La mala educación. Ideas que inspiraron el movimiento estudiantil en Chile. Santiago: Catalonia/CIPER, 2012.

Auguste, S.; Valenzuela, J. Do students benefit from school competition? The Chilean experience. 2004. Tesis (Doctoral Dissertation) - University of Michigan, Ann Arbor,2004.

BAll, S.; Youdell, D. Hidden privatization in public education. Londres: University of London, 2008.

Bauman, Z. Sobre la educación en un mundo líquido. Buenos Aires: Paidós, 2013.

Bellei, C. Expansión de la educación privada y mejoramiento de la educación en Chile. Evaluación a partir de la Evidencia. Revista Pensamiento Educativo, Santiago, v. 40, n. 1, p. 285-311, 2007. 
.; Contreras, D.; Valenzuela, J. Ecos de la revolución pingüina. Santiago: Universidad de Chile/UNICEF, 2010.

.; De Los Ríos, D.; Valenzuela, J. Evolución de la segregación socioeconómica de los estudiantes chilenos y su relación con el financiamiento compartido. Santiago: FONIDE/ MINEDUC, 2008.

.; GonzÁlez, C. Sostenibilidad del mejoramiento escolar impulsado por programas de asistencia técnica educativa. Revista Perspectiva Educacional, Valparaíso, v. 52, n. 1, p. 31-67, 2013.

.; Orellana, V. What does "Education Privatization" mean? Conceptual discussion and empirical review from Latina American cases. Santiago de Chile: Universidad de Chile, 2014. (Documento de Trabajo n. 14).

.; Trivelli, C. Apoyo público a escuelas privadas: casos internacionales y lecciones para Chile. Santiago de Chile: Universidad de Chile, 2014. (Documento de Trabajo n. 12). .; Valenzuela, J.; Vanni, X.; Contreras, D. Lo aprendi en la escuela. ¿Cómo se logran procesos de mejoramiento escolar? Santiago: Universidad de Chile/UNICEF, 2014. .; VAnni, X. The evolution of educational policy in Chile: 1980-2014. In: Schvartzman, S. (Ed.). Educ'ation in South America. New York: Bloomsbury, 2015. p. 179-200.

Canales, M.; Bellei, C.; Orellana, V. ¿Por qué elegir una escuela privada subvencionada? Sectores medios emergentes y elección de escuela en un sistema de mercado. Revista Estudios Pedagógicos, Valdivia, v. 42, n. 3, p. 89-109, 2016.

CARnoy, M. National voucher plans in Chile and Sweden. Did privatization reforms make for better education? Comparative Education Review, Chicago, v. 42, n. 3, p. 309-337, 1998. Castells, M. The information age. The power of identity. v. II. Malden: Blackwell, 1997. Снuвв, J.; Moe, T. Politics, markets and the organization of school. American Political Science Review, Cambridge, v. 82, n. 4, p. 1065-1087, 1988.

Cox, C. Política y Políticas educacionales en Chile 1990-2010. Revista Uruguaya de Ciencia Politica, Montevideo, v. 21, n. 1, p. 13-42, 2012.

Delanoy, F. Education reforms in Chile, 1980-1998: a lesson in pragmatism. Country Studies. Education Reform and Management Publication Series. v. I. n. I. Washington, D.C.: The World Bank, 2000.

Elacqua, G.; Santos, H. Preferencias reveladas de los proveedores de educación privada en Chile: el caso de la Ley de Subvención Escolar Preferencial. Revista Gestión y Politica Pública, v. 22, n. 1, p. 85-129, 2013.

Flores, C.; Carrasco, A.(Des)Igualdad de oportunidades para elegir escuela: preferencias, libertad de elección y segregación escolar. Santiago: Espacio Público, 2013.

Friedman, M. The Role of government in education. In: Solo, R.(Ed.). Economics and the Public interest. New Brunswick, N.J.: Rutgers University, 1955.

Gobierno de Chile. Ley n. 20.370. Establece la Ley General de Educación - LGE: Biblioteca del Congreso Nacional de Chile, 2009. Disponible en: <http://bcn.cl/1uvx5 >. Acceso en: 12 Sept. 2018. 
Lundströ̈m, U.; Parding, K. Teacher's experience with school choice: clashing logics in the Sweden educational system. Education. Research International, v. 2011, Article ID 869852, p. 1-10, 2011.

Maroy, C. Changes in regulation modes and social production of inequalities in education systems: a European comparison. Brussels: European Commission, 2004.

Patrinos, H.; Barrera-Osorio, F.; Guaqueta, J. The Role and impact of public private partnerships in education. Washington, D.C.: The World Bank, 2009.

Tокмan, A. Is private education better? Evidence from Chile. Santiago de Chile: Central Bank of Chile, 2002. (Documento de Trabajo n. 147).

Tooley, J. A market-led alternative for the curriculum: breaking the code. Londres: Institute of Education, 1993.

VAN Zanten, A. New modes of reproducing social inequality in education: the changing roles of parents, teachers, schools and educational policies. European Educational Research Journal, v. 4, n. 3, p. 155-169, 2005.

Verger, A.; Bonal, X.; Zancajo, A. What are the role and impact of public-private partnerships in education? A realist evaluation of the chilean education quasi-market. Comparative Education Revierw, v. 60, n. 2, p. 223-248, 2016.

Weinstein, J.; Muñoz, G.; Marfán, J. Liderar bajo presión: las estrategias gestionadas por los directores de escuelas para alcanzar los resultados comprometidos. In: Weinstein, J.; Muñoz, G. (Ed.). ¿¿Qué Sabemos de los Directores de Escuela en Chile? Santiago: Fundación Chile/CEPPE, 2013. p. 219-254.

Woods, P.; Bagley, C.; Glatter, R. School choice and competition: markets in the public interest. London: Routledge, 1998.

\section{SOBRE LOS AUTORES}

Juan Antonio Carrasco Bahamonde es magister en filosofía y magister en ciencias sociales por la Universidad de Chile (Chile). Investigador de la Universidad de Chile.

E-mail: carrasco661@gmail.com

Daniel Alonso Carrasco Bahamonde es magister en psicología social aplicada por la Universidad de Santiago (Chile). Investigador de la Universidad Tecnológica de Chile.

E-mail: danielcarrasco_41@yahoo.com

Recibido el 19 de junio de 2017 Aprobado el 10 de octubre de 2017 